Neurosurg Focus 21 (4):E7, 2006

\title{
Back and neck pain in triathletes
}

\author{
Alan T. Villavicencio, M.D., Sigita Burneikienė, M.D., Theresa D. Hernández, Ph.D., \\ AND JEFF THRAMANN, M.D.
} Boulder Neurosurgical Associates; and Department of Psychology and The Center for Neuroscience,
University of Colorado, Boulder, Colorado

\begin{abstract}
Object. As the sport of triathlon has continued to grow, increasing numbers of triathletes have presented in the neurosurgery clinics with various spinal disorders. This epidemiological study was undertaken to establish the lifetime incidence of neck and back pain, to gauge the prevalence of discogenic pain, and to identify risk factors among triathletes in the Boulder, Colorado, area.

Methods. A live online questionnaire was developed that was used to collect information about physical characteristics, training habits, athletic status, number of races completed, and back pain among triathletes. The incidence of cervical and/or lumbar discogenic back pain was defined according to the duration of symptoms for the most recent pain episode.

The lifetime incidence of low-back pain was $67.8 \%$, with $23.7 \%$ of cases possibly being discogenic in origin. The number of triathlons in which the respondents had participated and the presence of previous sports-related injuries were predictive of low-back pain ( $p=0.02$ and $p<0.00001$, respectively). The lifetime incidence of neck pain was $48.3 \%$, with $21.4 \%$ of cases being consistent with intervertebral disc involvement. The number of previous sports-related injuries was predictive of neck pain $(\mathrm{p}<0.00001)$, and a strong tendency toward neck pain was observed for athletes with more total years of participation in sports $(\mathrm{p}=0.06)$.

Conclusions. The two main risk factors for long-term spinal problems include sports-related injuries and overuse. The study results definitely support the influence of both mechanisms for low-back pain. Neck pain was associated with an injury event, and a strong (although not statistically significant) tendency toward neck pain was observed in respondents with overuse injuries.
\end{abstract}

\section{KEY WORDS - low-back pain • neck pain • athletic injury • sports medicine • triathlete}

$\mathrm{T}$ HE sport of triathlon was conceived in the early 1970s and consists of swimming, biking, and running, with each leg of the race a different distance. It gained worldwide recognition with the creation of the Ironman Triathlon in Hawaii, and was recently introduced as part of the Olympic games in Sydney, Australia, during the summer of 2000. Sports medicine specialists initially thought that triathlons would be associated with less overuse injury due to more balanced stress distribution in the musculoskeletal system. Unfortunately, this theory has proven deficient.

It has been found that triathletes tend to train more hours per week than any other group of single-sport athletes, which leads to a higher incidence of injury. ${ }^{12} \mathrm{~A}$ study performed shortly after the 1986 Hawaii Ironman Triathlon competition demonstrated that $91 \%$ of athletes had suffered at least one soft-tissue overuse injury during the previous year's training and that spine injuries were most common, with $72 \%$ of athletes reporting low-back pain or sciatica. ${ }^{17}$ Japanese triathletes were evaluated in another study, in which a 59\% lifetime (64\% in men and $41 \%$ in women) and $32 \%$ yearly (33\% in men and $29 \%$ in women) incidence of low-back pain were found. ${ }^{13}$ Most

Abbreviations used in this paper: BMI = body mass index; VAS = Visual Analog Scale. recent studies of triathletes are primarily focused on overuse or traumatic injuries, including back pain, ${ }^{4,17}$ and concern has been expressed by some authors that investigation of the long-term consequences of participation in this sport is needed.

Although the prevalence of neck pain in triathletes is not as high as low-back pain, ${ }^{13}$ it is surprising that neck overuse or traumatic injury was not even included in the analysis in the majority of published studies that were conducted to evaluate injuries in triathletes. ${ }^{4,6,11,17}$ In a study performed in recreational cyclists, up to $48.8 \%^{20,21}$ of cycling-related neck overuse injuries were reported, and it was the most common overuse injury encountered. ${ }^{21}$ Hill, et al., ${ }^{10}$ investigated factors that predispose individuals in the general population of those with neck pain to persistent symptoms. Cycling was found to be a strong risk factor for persistent neck pain, which these authors thought was related to posture, vibrations, a specific type of neck rotation, and overuse of the neck extensor muscle groups.

As the sport of triathlon has continued to grow during the past several years, increasing numbers of athletes with various spine conditions have been seen in neurosurgery clinics. Recurrent symptoms or persistent and chronic back pain that lasts longer than 3 months is more typically associated with degenerative disc disease, spondyloly- 
A. T. Villavicencio, et al.

sis, spondylolisthesis, injuries to the intervertebral disc, facet syndrome, or even fractures. ${ }^{1,9,19,22}$ A different approach is needed when treating athletes because of their high levels of activity and expectation of returning to sports competitions. Fortunately, the majority of sportsrelated back problems resolve with conservative, nonsurgical management. Although surgical intervention is sometimes the fastest route to get the patient-athlete back into active sports, it may not be the best solution, and management of the injury should be decided on an individual basis. Extensive conservative measures should be exhausted before considering surgery.

This epidemiological study was undertaken to establish the lifetime incidence of neck and back pain among triathletes in the Boulder, Colorado, area. The secondary objectives were to identify risk factors associated with the occurrence of neck and back pain and to estimate the probability of discogenic problems in triathletes.

\section{Online Questionnaire}

\section{Clinical Material and Methods}

A self-administered online questionnaire was developed, which included three groups of questions. A list of approximately 4000 email addresses obtained from a local race director was used to send a generic message to triathletes. This short, one-sentence message was included in a group of multiple other short messages from the race director and included a direct Web link to the live, online survey. Once the survey had been completed, the results were automatically sent to a secure email address for statistical analysis. The email list had been generated over a more than 10-year time period, and many of the addresses were probably no longer in use; it is not known how many of them were valid. It is also unknown how many triathletes actually read all of the messages from the race director or were even aware of the link.

In the first part of the questionnaire, we collected information about the respondents' physical characteristics, training habits, athletic status, and number of races completed. The second part consisted of questions regarding low-back pain, including frequency, duration, and intensity of both the overall symptoms and of the most recent pain episode, relationship of the pain to sporting events, athlete's age at first pain episode, incidence of injuries, medical care received, and sciatica symptoms. The third part included similar questions about neck pain.

There is no consensual definition of back pain that would clearly distinguish nonspecific pain and back pain with a discogenic origin. Therefore, the specificity or severity of the cervical and/or lumbar back pain in our study was defined according to the duration of symptoms for the most recent pain episode: fewer than 7 days (acute), fewer than 3 months (subacute), and more than 3 months (chronic). This type of classification was described by Mooney ${ }^{15}$ and is based on the duration of the episode and the hypothesis that back pain is mainly discogenic. According to this classification, back pain that lasts for fewer than 7 days is generally related to tendon, ligament, and fascia injuries, and thus has the highest potential for spontaneous recovery. Subacute pain that persists longer than 7 days but fewer than 3 months may also involve facet joints. Chronic back pain typically lasts longer than 3 months and is
TABLE 1

Distribution of 87 triathletes by sex and age groups

\begin{tabular}{cccc}
\hline \hline Age Group & Men & Women & Total No. (\%) \\
\hline $18-24$ & 1 & 6 & $7(8.0)$ \\
$25-29$ & 5 & 13 & $18(20.7)$ \\
$30-34$ & 3 & 12 & $15(17.2)$ \\
$35-39$ & 11 & 10 & $21(24.1)$ \\
$40-44$ & 3 & 7 & $10(11.5)$ \\
$45-49$ & 2 & 6 & $8(9.2)$ \\
$50-54$ & 2 & 2 & $4(4.6)$ \\
$55-59$ & 2 & 0 & $2(2.3)$ \\
$60-64$ & 1 & 0 & $1(1.2)$ \\
$65-69$ & 1 & 0 & $1(1.2)$ \\
total & 31 & 56 & 87 \\
\hline
\end{tabular}

more likely to be disc related. Each of these categories also refers to radiculopathy symptoms. As mentioned, acute pain has the highest potential for spontaneous recovery with or without treatment. Subacute and chronic back pain involve more significant morphological and structural damage, and thus conservative treatment is indicated, which includes medications (antiinflammatory, muscle relaxant, and oral steroid drugs), physical therapy, and injections. Some athletes, especially those in the chronic pain group, may benefit from surgical interventions.

Based on the respondent's own perspective, athletic status was classified as elite, intermediate, and beginner. Athletes were asked to answer questions about the number of injuries they had suffered. An injury was defined as an acute event that led the individual to seek medical care, medications, or to cease training for at least 1 day.

The following scale and scoring system was used to assess the frequency of back pain episodes: 1, back pain once a year; 2 , once in 6 months; 3 , once in 3 months; 4 , once a month; 5 , once a week; and 6 , constant. Pain intensity was established using the VAS. On the basis of the responses we have received, a database was created and data were analyzed. Logistic regression analysis was used to determine any statistically significant associations or correlations between back pain and multiple physical, training, and competition-related factors.

\section{Study Population}

\section{Results}

Our study population consisted of triathletes who were living and training in the Boulder, Colorado, area. Eightyseven questionnaires were received in response to the approximately 4000 emails that were generated. Again, many of these addresses were probably duplicates or were obsolete because athletes had changed their email addresses over the years. Of the 87 respondents, $31(35.6 \%)$ were male and $56(64.4 \%)$ were female. The mean age of all athletes was 36.1 years (range 20-68 years); 34.1 years (range 20-53 years) for women and 39.8 years (range 24-68 years) for men. The distribution by age groups and sex is presented in Table 1 . There were six $(6.9 \%)$ elite, 65 $(74.7 \%)$ intermediate, and $16(18.4 \%)$ beginner triathletes.

\section{Training Patterns}

The mean number of triathlons (including Iron distance events) and other running races in which the respondents 
had participated was 22.4 (range 1-150) for all athletes or 75.6, 25, and 6.3 races for elite, intermediate, and beginner triathletes, respectively. The mean combined training time was 12.5 hours per week. Swimming, biking, and running accounted for 22,47 , and $31 \%$ of training time, respectively (Table 2). Fifty-five triathletes (63\%) supplemented their training with weight lifting for an average of 1.5 hours per week (range 0.25-6 hours). Forty-four triathletes $(51 \%)$ were participating in other kinds of sports (for example, skiing and basketball) an average of 3.4 hours per week (range 1-18 hours). All included, the total mean training time was 15.2 hours per week (range 5-30 hours).

\section{Low-Back Pain}

The lifetime incidence of low-back pain was $67.8 \%$ (59 of 87 respondents); 48 (81.4\%) of 59 athletes indicated that their lumbar back pain was related to a sports injury. Weight lifting, running, cycling, and other sports were responsible for $4.2,22.9,35.4$, and $39.6 \%$ of all sports-related low-back injuries, respectively.

The duration of the most recent low-back pain episode was fewer than 7 days in $37(62.7 \%)$ of 59 triathletes. Eight $(13.6 \%)$ of the 59 had a pain episode that lasted fewer than 3 months, and in the other $14(23.7 \%)$ the pain episode lasted more than 3 months. Table 3 illustrates the demographic data, training patterns, and low-back pain characteristics; this information is categorized according to the duration of the lumbar back pain during its last occurrence.

Triathletes With Acute Lumbar Pain. This group of patients noted that their low-back pain lasted fewer than 7 days and therefore was possibly related to tendon, ligament, and fascia injuries without major structural changes. As expected, this was the largest group in our study and consisted of 37 triathletes $(62.7 \%)$. However, even in this group, nine athletes (24.3\%) noted sciatica symptoms. Only seven patients (18.9\%) in this group had never received treatment for their back pain.

The mean age of the triathletes in this group was 36.3 years (range 24-68 years). Their weekly training schedule consisted of 14.9 hours on average (range 6-30 hours). They had participated in 24.1 running races and triathlons on average (range 1-150). Although only nine (24.3\%) discontinued training to alleviate their symptoms, all but two thought that their back pain was sports related. The

\section{TABLE 2}

Overall distribution of weekly training time in 87 triathletes according to self-reported athletic status*

\begin{tabular}{llll}
\hline \multicolumn{1}{c}{ Sport } & \multicolumn{1}{c}{ Elite } & Intermediate & \multicolumn{1}{c}{ Beginner } \\
\hline swimming & $4.2(3-6)$ & $2.7(1-9)$ & $2.7(1-4.5)$ \\
biking & $8.9(4-15)$ & $5.8(1-15)$ & $4.6(2-6.5)$ \\
running & $4.8(2.5-8)$ & $3.9(1-7)$ & $3.6(2-6.5)$ \\
weight lifting & $1.0(0-2)$ & $1.0(0-6)$ & $0.6(0-3)$ \\
other & $1.5(0-3)$ & $1.9(0-18)$ & $0.8(0-3)$ \\
total no. & 6 & 65 & 16 \\
$\quad$ of athletes & & & \\
\hline
\end{tabular}

* Except for number of athletes, values are given as the mean number of hours, with the range in parentheses. mean number of injuries for this group was 1.2 (range $0-7)$. An injury was defined as an acute event that led the athlete to seek medical care, medications, or to cease training for at least 1 day.

Triathletes With Subacute Lumbar Pain. Eight athletes $(13.6 \%)$ were included in this group. Their most recent back pain episode lasted more than 7 days but fewer than 3 months. Only one patient in this group had sciatica symptoms. All triathletes in this group received conservative treatment, and none underwent surgery. The mean age in this group was 36.2 years (range $25-55$ years). The total weekly training time was 17.3 hours (range 11-24 hours). Athletes in this group had participated in 24.2 triathlons on average (range 3-54). Four of eight respondents thought that their low-back pain was sports-related, and all of them stopped training while in pain. The mean number of sports-related injuries for this group was 1.5 (range 0-5).

Triathletes With Chronic Lumbar Pain. Fourteen (23.7\%) of 59 triathletes noted that the most recent low-back pain episode lasted more than 3 months and, according to Mooney ${ }_{1}^{15}$ it could be presumed that more permanent morphological abnormalities had occurred that involved intervertebral discs. Nine $(64.3 \%)$ of these 14 patients also reported sciatica symptoms. All 14 athletes in this group received some conservative treatment for their low-back pain; seven had physical therapy, and others underwent various treatments including medications, massage, chiropractic manipulations, and so on. One patient in this group underwent surgery. The mean age for this group was 36.9 years (range $22-56$ years). The total weekly training time was 15 hours (range 5-25 hours), which was comparable to the other groups (Table 3 ) as well as to the triathletes with no low-back symptoms. On average, respondents in this group had participated in 21.4 triathlons and marathons (range 2-95), compared with 20.1 (range 2-65) in the asymptomatic triathletes $(\mathrm{p}=0.8, \mathrm{t}$-test $)$ and 24.1 (range 1-150) for the athletes whose lumbar pain lasted more than 7 days and fewer than 3 months. Nine (64.3\%) of 14 triathletes thought that their low-back pain was related to sports, and the mean number of sports-related injuries was 1.6 (range $0-5$ ) in this group. Ten $(71.4 \%$ ) of 14 stopped training while they were in pain.

\section{Statistical Analysis}

Logistic regression analysis was performed to determine if any specific factors were predictive of low-back pain incidence. There was no statistically significant correlation between low-back pain and age $(\mathrm{p}=0.5)$, sex $(\mathrm{p}=$ $0.1)$, athletic status $(\mathrm{p}=0.4), \mathrm{BMI}(\mathrm{p}=0.7)$, or training duration $(p=0.9)$. A strong tendency toward low-back pain was observed for the respondents with more total years of participation in sports $(\mathrm{p}=0.06)$, and the number of triathlons in which they had competed was predictive of low-back pain $(p=0.02)$. A very strong correlation was observed between the number of previous sports-related injuries and the presence of low-back pain ( $\mathrm{p}<0.00001)$.

\section{Neck Pain}

The lifetime incidence of neck pain was slightly lower than that of low-back pain; $42(48.3 \%)$ of the 87 triathletes who responded. Twenty-six (61.9\%) of 42 athletes indi- 
TABLE 3

Demographic data and selected low-back pain characteristics in three low-back pain groups*

\begin{tabular}{lccc}
\hline \hline & \multicolumn{2}{c}{ Duration of Low-Back Pain Episode } \\
\cline { 2 - 4 } \multicolumn{1}{c}{ Characteristic } & Acute & Subacute & Chronic \\
\hline no. of athletes (\%) & $37(62.7)$ & $8(13.6)$ & $14(23.7)$ \\
mean age in yrs & 36.3 & 36.2 & 36.9 \\
range & $24-68$ & $25-55$ & $22-56$ \\
M/F ratio & $15: 22$ & $4: 4$ & $5: 9$ \\
mean pain duration in yrs & 8.7 & 14.1 & 12.0 \\
range & $3-15$ & $0.25-40$ & $0.75-30$ \\
mean pain intensity & 4.6 & 6.2 & 5.6 \\
$\quad$ (VAS score) & $1-10$ & $3-9$ & $3-10$ \\
range & 3.7 & 4.3 & 5.6 \\
mean frequency score $\dagger$ & $1-6$ & $1-6$ & $3-6$ \\
range & 27.2 & 22.4 & 28.2 \\
age in yrs at 1st back & & & \\
pain episode & $14-65$ & $13-35$ & $11-53$ \\
range & 1.2 & 1.5 & 1.6 \\
mean no. of injuries & $0-7$ & $0-5$ & $0-5$ \\
range & 14.9 & 17.3 & 15.0 \\
total training time (hrs/wk) & $6-30$ & $11-24$ & $5-25$ \\
range & 24.1 & 24.2 & 21.4 \\
mean no. of triathlons & $1-150$ & $3-54$ & $2-95$ \\
range & & & \\
\hline
\end{tabular}

* Acute, fewer than 7 days; subacute, more than 7 days and fewer than 3 months; chronic, more than 3 months.

$\dagger$ The mean pain frequency score was assessed based on a scale of 1 to 6 (6, constant pain; 1 , once/year).

cated that their neck pain was sports related and 17 $(65.4 \%)$ of 26 thought that it was associated with cycling. The duration of the latest neck pain episode was fewer than 7 days in $28(66.7 \%)$ of 42 athletes, it lasted fewer than 3 months in five $(11.9 \%)$ of the 42 , and more than 3 months in the other nine $(21.4 \%)$ who noted neck pain. Table 4 illustrates the demographic data, training patterns, and neck pain characteristics according to the duration of the most recent neck pain episode.

Triathletes With Acute Cervical Pain. This was the largest group in our study and consisted of triathletes in whom the latest episode of neck pain lasted fewer than 7 days. According to the Mooney classification, ${ }^{15}$ this type of neck pain has the highest potential for spontaneous recovery. This group consisted of 28 respondents and made up $66.7 \%$ of all athletes who reported neck pain. Only one athlete in this group (3.6\%) noted radicular symptoms. Nine respondents $(32.1 \%)$ had not received treatment for their neck pain; the rest had undergone some type of conservative treatment, with massage being prevalent $(57.1 \%)$. The mean age in this group was 36.8 years (range 20-68 years). The weekly training schedule consisted of 15.1 hours (range 6-30 hours). The mean number of triathlons and running races in which the athletes had participated was 19 (range 1-91) for this group. The majority of triathletes $(67.9 \%)$ in this group thought that their neck pain was sports related, but only six $(21.4 \%)$ discontinued their training while in pain. The mean number of injuries was 2.3 (range 0-20).

Triathletes With Subacute Cervical Pain. Only five athletes who responded noted that their neck pain lasted longer than 7 days but fewer than 3 months. Only one re- spondent in this group had radicular symptoms. All but one had received some type of conservative treatment for their neck pain. The mean age was 32.8 years (range 28-38 years). The total weekly training time was 15.9 hours on average (range 10.5-32 hours), and it did not vary significantly from the other groups (Table 4 ). The difference was not quite statistically significant for the number of triathlons and running races in which the athletes had participated ( $\mathrm{p}=0.059$, t-test) when compared with the respondents who reported acute or chronic cervical pain. Four of the five triathletes thought that their neck pain was sports-related, and they stopped training while in pain. The mean number of injuries for this group was 2 (0-5).

Triathletes With Chronic Cervical Pain. Nine (21.4\%) of 42 athletes who noted neck pain in this study stated that the most recent pain episode lasted longer than 3 months. Based on the Mooney theory, this pattern may suggest that the intervertebral disc is the most likely source of their pain. All athletes in this group reported constant pain. A scale and scoring system used to assess the frequency of back pain episodes is described in Clinical Material and Methods.

The mean age of respondents when they experienced their first neck pain episode was 26.8 years (range 10-40 years). Three athletes $(33.3 \%)$ had radicular symptoms, but only one stopped training while in pain. All athletes in this group underwent some kind of conservative treatment, such as medications and/or physical therapy. The mean age in this group was 34.8 years (range 22-53 years). The average total weekly training time was 13.1 hours (range 9-20 hours). On average, athletes participated in 17.6 triathlons and other running races (range 3-50). Only three $(33.3 \%)$ of nine athletes thought that their pain was related to sports, and all of them noted previous sports-related injuries.

TABLE 4

Demographic and selected neck pain characteristics in three neck pain groups*

\begin{tabular}{|c|c|c|c|}
\hline \multirow[b]{2}{*}{ Characteristic } & \multicolumn{3}{|c|}{ Duration of Neck Pain Episode } \\
\hline & Acute & Subacute & Chronic \\
\hline no. of athletes (\%) & $28(66.7)$ & $5(11.9)$ & $9(21.4)$ \\
\hline mean age in yrs & 36.8 & 32.8 & 34.8 \\
\hline range & $20-68$ & $28-38$ & $22-53$ \\
\hline $\mathrm{M} / \mathrm{F}$ ratio & $13: 15$ & $3: 2$ & $3: 6$ \\
\hline mean pain duration in yrs & 10.0 & 8.6 & 8.7 \\
\hline range & $0.5-50$ & $2-13$ & $0.5-20$ \\
\hline $\begin{array}{l}\text { mean pain intensity } \\
\text { (VAS score) }\end{array}$ & 4.0 & 6.2 & 6.5 \\
\hline range & $1-8$ & $4-8$ & $4-9$ \\
\hline mean frequency score $\dagger$ & 3.6 & 4.4 & 6.0 \\
\hline range & $1-6$ & $3-6$ & 6.0 \\
\hline $\begin{array}{l}\text { age in yrs at } 1 \text { st back } \\
\text { pain episode }\end{array}$ & 27.3 & 22.8 & 26.8 \\
\hline range & $15-55$ & $16-32$ & $10-40$ \\
\hline mean no. of injuries & 2.3 & 2.0 & 0.75 \\
\hline range & $0-20$ & $0-5$ & $0-2$ \\
\hline total training time (hrs/wk) & 15.1 & 15.9 & 13.1 \\
\hline range & $6-30$ & $10.5-32$ & $9-20$ \\
\hline mean no. of triathlons & 19.0 & 36.2 & 17.6 \\
\hline range & $1-91$ & $17-54$ & $3-50$ \\
\hline
\end{tabular}

* See Table 3 footnote for explanation of terminology. 


\section{Statistical Analysis}

Logistic regression analyses were performed to determine if any of the factors were predictive of the incidence of neck pain. There was no statistically significant correlation between cervical pain and age $(\mathrm{p}=0.8)$, athletic status $(p=0.2)$, BMI $(p=0.2)$, training duration $(p=0.9)$, or number of races $(\mathrm{p}=0.2)$. Whereas statistical significance with respect to neck pain and sex $(\mathrm{p}=0.07)$ could not be demonstrated, a strong tendency was observed for neck pain being related to sex, with $19(74.2 \%)$ of the 31 men reporting neck pain compared with 23 (41.1\%) of the 56 women in our study. A strong tendency was also observed for neck pain depending on the total years of participation in sports $(\mathrm{p}=0.06)$. An extremely strong correlation was observed between neck pain and the number of previous sports-related injuries $(\mathrm{p}<0.00001)$.

\section{Discussion}

The majority of neck and back pain in our study lasted fewer than 7 days, from which we inferred mainly soft-tissue and ligament involvement. Triathletes who responded to our questionnaire appeared to experience frequent longer-term discomfort, which may suggest a high rate of discogenic pain. Of all athletes who had experienced neck or low-back pain in their lifetime, more than $20 \%$ noted that the most recent pain episode lasted longer than 3 months. Radiculopathy symptoms were very common, with 64.3 and $33.6 \%$ of triathletes reporting them in the chronic lowback and neck pain groups, respectively. Radicular symptoms do not appear to cause athletes to stop training. It is likely that triathletes simply change their routine to involve more swimming or biking and less running, or vice versa, depending on what exacerbates their symptoms. This aspect was not evaluated in our study and deserves further investigation.

In addition, the intensity of back pain symptoms revealed in this study is troubling. The mean VAS score was 6.5 for neck pain and 5.6 for low-back pain. A mean frequency score of up to 5.6 for low-back pain and up to 6 for neck pain ( 6 was the highest score, representing constant pain) was reported for the triathletes who suffered chronic back pain. The scale and scoring system that we used to assess the frequency of back pain episodes is described in Clinical Material and Methods (please also see Tables 3 and 4, mean frequency score). Manninen and Kallinen ${ }^{13}$ reported that $19 \%$ of all low-back pain episodes lasted longer than 3 months among the Japanese triathletes in their study. They indicated that "it was a clear danger sign of potential future and possibly severe long term disability." We believe that the prognosis for this situation is not necessarily that grave. Much more optimistic prognoses could be made for these athletes, given the new technologies available for spine surgery, including the development of minimally invasive techniques, artificial discs, interspinous implants, and dorsal dynamic stabilization systems.

One risk factor for back pain is related to the natural history of degenerative changes in the general population. The belief that these changes should increase with age has been supported by several publications., ${ }^{3,18}$ In one study comparing the pathogenesis of low-back pain, an $11 \%$ incidence of discogenic back pain was reported in young athletes compared with $48 \%$ in an adult general population. ${ }^{14}$ However, there were no statistically significant correlations observed between age and back pain in our small group of triathletes, even though $26(30 \%)$ of our 87 respondents were 40 years of age or older. This finding can be attributed to the fact that the major risk of back pain was related to the incidence of injury, which could occur at any age.

Sex, athletic status, BMI, and training duration were not predictive of back pain in our study. Thus, back pain appears to be rather equally distributed across the population of triathletes, with no single demographic factor being significantly more associated with risk than another. Although there was also a strong trend for total years of participation in sports to predict pain, the number of triathlons in which the respondents had competed and the presence of previous sports-related injuries were the only factors that demonstrated a statistically significant association with the lifetime incidence of low-back pain. Sports medicine-related literature generally supports the theory that back pain is most frequently caused by traumatic injuries and overuse. Our low-back pain study results support this hypothesis by demonstrating statistically significant correlations between back pain and the number of previous sports-related injuries or number of races completed. On the other hand, we could not observe a definite correlation between neck pain and the total number of races completed or years in sports, although a strong tendency toward significance was observed for the latter.

On the basis of our results, the triathletes at greatest risk of chronic neck and low-back pain included those with higher participation rates in competition (lumbar pain), total years in sports (neck pain), and especially a history of injury (both neck and low-back pain). In some ways this seems counterintuitive: one could speculate that greater fitness levels would be required to participate in more events, and that greater fitness levels would not be associated with physical pain. It may also be the case that participating in more events, regardless of fitness level, could result in a higher number of injuries due to overuse, leading to more pain. When left untreated, overuse injuries are thought to lead to a cumulative overload and consequently to spine discogenic pain. It was demonstrated in a prospective study that athletes who reported previous low-back injury were three times more predisposed to sustain a low-back injury in the following year. ${ }^{8}$ Previous neck trauma has also been shown to be predictive of neck pain. ${ }^{5}$ This resultant self-perpetuating cycle could be specifically related to the demands of endurance sports in general, or to the multisport nature of the triathlon, which requires a higher number of weekly training hours.

Egermann, et al., ${ }^{6}$ studied the incidence of injuries during the Ironman Europe 2000 competition, and they specifically looked at previous years of training for the participants. Most of the chronic pain (for example, injuries to the knee and the Achilles tendon) was associated with overuse injury, except for back pain. Athletes in this study had a $31.2 \%$ rate of chronic low-back pain compared with a $26.8 \%$ rate of injury-associated back pain. Those who trained for longer than 20 hours per week experienced more chronic back pain than the others $(\mathrm{p}=0.002)$. Although we have demonstrated an association between low-back pain and the number of triathlons and running 
races in which the athletes have competed $(p=0.02)$, an even stronger correlation was demonstrated for the number of sports-related injuries $(\mathrm{p}<0.00001)$. A higher incidence of low-back or neck pain was not associated with training duration in our study.

There is a high lifetime incidence of back pain-associated disability in the general population in developed countries. ${ }^{7,19}$ A lifetime incidence of up to $80 \%$ has been reported for low-back pain. We report a lifetime incidence of $67.8 \%$ for low-back and $48.3 \%$ for neck pain in our small group of triathletes. This incidence is quite high, and our triathletes are slightly older than those who participated in the other studies (36.1 years overall [range 20-68 years]; 34.1 years for women [range 20-53 years] and 39.8 years for men [range 24-68 years]). There is a possibility that the lifetime incidence could be overestimated in our study.

Although it is debatable whether the incidence of back pain is lower or higher in athletes, the morbidity associated with such problems is quite clear. ${ }^{1}$ Whereas spinal anatomy and physiology may be the same for the nonathlete as for the athlete, the forces brought to bear on the spine in endurance sports like triathlons are in theory much greater than those experienced by nonathletes, and may increase the strain and the vulnerability of the spine or surrounding tissue to damage. ${ }^{19}$ Training duration requirements in triathletes may result in chronic spine pain that is discogenic in origin.

In our study, some of the triathletes reported that their back pain was not sports related, but overuse injuries and intense repetitive loads during training and competitions may well exacerbate it. Early back pain symptoms should not be ignored, even if they last for fewer than 7 days and the athlete recovers spontaneously. The origins of injuries in triathletes should be assessed to modify the cycle of overuse-associated injury and to increase training time devoted to flexibility and muscular balance. In addition, a temporary modification of training technique has been suggested by some authors (that is, more time devoted to swimming). ${ }^{17}$

Minimizing disability associated with spine pain is as important in the athlete as in the nonathlete. Nevertheless, the most efficacious methods for doing so may be distinct for each group. A strong functional rehabilitation program $^{2,16,19,22,23}$ tailored to the athlete (for example, one that takes into account their high level of activity and endurance as well as the strong motivation to return to training and competition) could effectively address early backrelated problems and help prevent the long-term consequences.

\section{Limitations of the Study}

The present study has several limitations. First, the group surveyed came primarily from the Boulder, Colorado, area, and may not be representative of triathletes in general, even though a large number of triathletes choose to train in this region because of its higher elevation and bike-friendly roads. Second, we had a low response rate to our questionnaire, which also may influence generalizability. We asked all triathletes to respond, regardless of the presence or absence of symptoms, but it is reasonable to assume that the athletes who have back pain symptoms would be more likely to respond than asymptomatic individuals. This distinction means that the lifetime incidence of back and neck pain may be overestimated in our study. However, that should not have affected our attempt to identify the prevalence of possible discogenic back pain that might require intensive multidisciplinary rehabilitation, conservative treatment, or surgical intervention.

Although data were collected prospectively, athletes filled out the survey in a retrospective fashion. As a result, these data may not be as accurate as those gathered in a prospective study. Moreover, these results are based on self-reported information, and no verification was available from an independent source.

Many more female triathletes $(64.4 \%)$ responded to the questionnaire compared with the number in other published epidemiological studies of triathletes, and therefore our sample was not well balanced. The ratio of female triathletes is generally slightly above $20 \%$ in the other studies. ${ }^{4,11,13,17}$ Also, our study results demonstrated that a strong tendency toward neck pain is more prevalent in male athletes (of all athletes, neck pain was present in $74.2 \%$ of men compared with $41.1 \%$ of women), which contradicts the previously reported results that the odds of female cyclists developing neck and shoulder pain were up to two times higher than for male cyclists. ${ }^{9}$ Because the significance of the difference is unknown, further evaluation is needed to assess these findings.

\section{Conclusions}

The lifetime incidence of neck and low-back pain was 48.7 and $67.8 \%$, respectively, in the triathletes in this study. The two main mechanisms of long-term pain, possibly implying discogenic pain, include sports-related injuries and overuse. Our study results definitely support the influence of both mechanisms for low-back pain. The incidence of neck pain was associated with an injury event, and a strong tendency toward neck pain was observed with overuse injuries.

\section{Disclosure}

This work was supported by the Justin Parker Neurosurgical Research Foundation.

\section{References}

1. Bono CM: Low-back pain in athletes. J Bone Joint Surg Am 86:382-396, 2004

2. Brennan GP, Fritz JM, Hunter SJ, Thackeray A, Delitto A, Erhard RE: Identifying subgroups of patients with acute/subacute "nonspecific" low back pain: results of a randomized clinical trial. Spine 31:623-631, 2006

3. Burdorf A, Sorock G: Positive and negative evidence of risk factors for back disorders. Scand J Work Environ Health 23: 243-256, 1997

4. Collins K, Wagner M, Peterson K, Storey M: Overuse injuries in triathletes. A study of the 1986 Seafair Triathlon. Am J Sports Med 17:675-680, 1989

5. Croft PR, Lewis M, Papageorgiou AC, Thomas E, Jayson MIV, Macfarlane GJ, et al: Risk factors for neck pain: a longitudinal study in the general population. Pain 93:317-325, 2001

6. Egermann M, Brocai D, Lill CA, Schmitt H: Analysis of injuries in long-distance triathletes. Int J Sports Med 24:271-276, 2003 
7. George SZ, Delitto A: Management of the athlete with low back pain. Clin Sports Med 21:105-120, 2002

8. Greene HS, Cholewicki J, Galloway MT, Nguyen CV, Radebold A: A history of low back injury is a risk factor for recurrent back injuries in varsity athletes. Am J Sports Med 29: 795-800, 2001

9. Harvey J, Tanner S: Low back pain in young athletes: a practical approach. Sports Med 12:394-406, 1991

10. Hill J, Lewis M, Papageorgiou AC, Dziedzic K, Croft P: Predicting persistent neck pain: a 1-year follow-up of a population cohort. Spine 29:1648-1654, 2004

11. Korkia PK, Tunstall-Pedoe DS, Maffulli N: An epidemiological investigation of training and injury patterns in British triathletes. Br J Sports Med 28:191-196, 1994

12. Levy CM, Kolin E, Berson BL: The effect of cross training on injury incidence, duration and severity (part 2). Sports Med Clin 3:1-8, 1986

13. Manninen JS, Kallinen M: Low back pain and other overuse injuries in a group of Japanese triathletes. Br J Sports Med 30: 134-139, 1996

14. Micheli LJ, Wood R: Back pain in young athletes: significant differences from adults in causes and patterns. Arch Pediatr Adolesc Med 149:15-18, 1995

15. Mooney V: The classification of low back pain. Ann Med 21: 321-325, 1989

16. Nelson BW, Carpenter DM, Dreisinger TE, Mitchell M, Kelly $\mathrm{CE}$, Wegner JA: Can spinal surgery be prevented by aggressive strengthening exercises? A prospective study of cervical and lumbar patients. Arch Phys Med Rehabil 80:20-25, 1999

17. O'Toole ML, Hiller WD, Smith RA, Sisk TD: Overuse injuries in ultraendurance triathletes. Am J Sports Med 17:514-518, 1989

18. Ozguler A, Leclerc A, Landre MF, Pietri-Taleb F, Niedhammer I: Individual and occupational determinants of low back pain according to various definitions of low back pain. J Epidemiol Community Health 54:215-220, 2000

19. Trainor TJ, Trainor MA: Etiology of low back pain in athletes. Curr Sports Med Rep 3:41-46, 2004

20. Weiss BD: Nontraumatic injuries in amateur long distance bicyclists. Am J Sports Med 13:187-192, 1985

21. Wilber CA, Holland GJ, Madison RE, Loy SF: An epidemiological analysis of overuse injuries among recreational cyclists. Int J Sports Med 16:201-206, 1995

22. Woolf SK, Glaser JA: Low back pain in running-based sports. South Med J 97:847-851, 2004

23. Young JL, Press JM, Herring SA: The disc at risk in athletes: perspectives on operative and nonoperative care. Med Sci Sports Exerc 29:S222-S232, 1997

Manuscript received July 18, 2006.

Accepted in final form September 11, 2006.

Address reprint requests to: Alan T. Villavicencio, M.D., Boulder Neurosurgical Associates, 1155 Alpine Avenue, Suite 320, Boulder, Colorado 80304. email: atv@ bnasurg.com. 